\title{
Critical review of propeller performance scaling methods, based on model experiments and numerical calculations
}

\author{
Tomasz Bugalski, Ph.D., \\ Ship Design and Research Centre CTO SA, Poland, \\ Heinrich Streckwall, Ph.D., \\ Hamburg Ship Model Basin, Germany, \\ Jan A. Szantyr, Prof., \\ Gdansk University of Technology, Poland
}

\section{ABSTRACT}

\begin{abstract}
The article presents the results of experimental and numerical investigation of propeller scale effects, undertaken in co-operation of the Hamburg Ship Model Basin (HSVA), Germany, and Ship Design and Research Centre (CTO SA), Poland. The objective of the investigation was to test the adequacy of the methods currently used to account for the propeller scale effect and to develop possible improvement of the methods. HSVA has conducted model experiments in the large cavitation tunnel together with panel method and CFD calculations. CTO SA has performed model experiments in the towing tank, together with lifting surface and CFD calculations. Both institutions have suggested different new approaches to the problem and different new procedures to account for the propeller scale effects. In the article the procedures are presented together with the description of the underlying experimental and theoretical research.
\end{abstract}

Keywords: propeller scale effects; open water tests; computational fluid dynamics

\section{INTRODUCTION}

Despite a rapid development of the computational fluid dynamics (CFD) the open water model experiments remain the basic source of information about the hydrodynamic characteristics of ship propellers. This information is later used for determination of the powering performance of real, full scale ships. As the complete hydrodynamic similarity between model experiments and full scale operation of propellers cannot be achieved, the appropriate methods for correction of the so called scale effect had to be developed and implemented in order to convert the hydrodynamic characteristics of a propeller model into their full scale equivalent. The differences between the model and full scale propeller characteristics arise mainly due to a marked difference in model and full scale Reynolds numbers, hence they are connected to viscous flow effects in both scales. The differences are illustrated in Fig. 1, showing the scale effect on propeller efficiency. The ideal (inviscid) efficiency does not depend on scale, therefore it is shown as a horizontal line. The real (viscous) flow around propeller model and full scale propeller exhibits pronounced scale effects. Accurate determination of the scale effects is the subject of research described in this paper.

Scale effects may be studied in experimental and numerical way. Suitably controlled experiments in model scale are relatively easy, but their full scale equivalents are almost impossible to conduct. Therefore, the CFD and other numerical methods may be helpful in studying the problem.
The research described in this paper has been undertaken by two ship hydrodynamic centres, namely the Hamburg Ship Model Basin (HSVA), Germany, and Ship Design and Research Centre (CTO SA), Gdansk, Poland. The joint project included model experiments conducted both in cavitation tunnels and towing tanks, together with CFD and lifting surface/surface panel potential flow calculations. The objective was to assess the adequacy of currently used methods for scale effect corrections to the geometry of contemporary ship propellers and, if possible, to suggest modifications of these methods.

\section{RESEARCH AT THE HAMBURG SHIP MODEL BASIN (HSVA)}

\section{Introductory considerations}

Usually the model - test - based prediction of the power at the full scale propeller involves tests in two Reynolds number ranges. In the ship self-propulsion mode the model propeller has to obey Froude similarity and appropriately defined Reynolds numbers $\mathrm{Re}_{0,7}$ are around $5^{*} 10^{5}$ (see below for definition). In addition to support the power prediction process open water tests are performed in the towing tank at moderate Reynolds numbers $\left(\operatorname{Re}_{0.7} \approx 1^{*} 10^{6}\right)$. In both cases there exists a Reynolds number range characterized by a mixture of turbulent and laminar flow on the propeller blade surface (laminar from the leading edge to a certain transition range and turbulent after switching). 


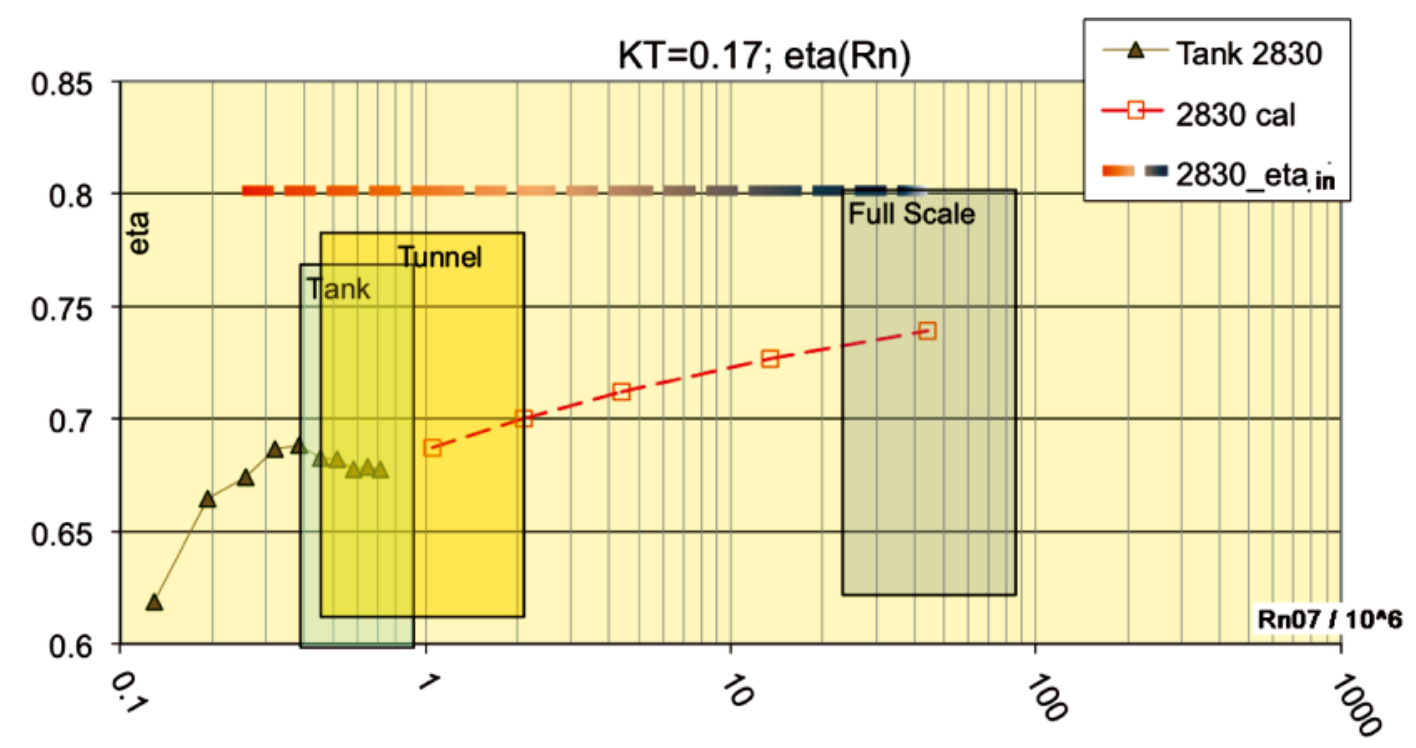

Fig. 1. Example of scale effect on propeller open-water efficiency, showing the ideal (inviscid) efficiency and real (viscous) efficiency for model tank, cavitation tunnel and full scale

Scaling procedures for propeller efficiency are to provide a good estimation for surface friction effects. In any scale they have to divide the propeller performance into two contributions. One part is valid under the absence of surface friction and thus is only counting forces normal to the propeller blade surface. For simplicity the low-order scaling methods consider this (dominating) share as not influenced by the Reynolds number. To arrive at the other share one has to estimate tangentially directed blade surface forces. The local magnitude of such forces may give a complex surface pattern, especially if the above mentioned transition results in a more or less equal share of laminar and turbulent flow on the blade.

A strip method was considered as a good compromise between treating surface friction in a reasonably detailed way and being still robust and quick. Accordingly, such a procedure was to be developed up to a quality and robustness which would allow using it as a new standard method and replace HSVA's former scaling procedure according to Lerbs/Meyne [5].

\section{Principles of a new strip method}

The quality of a scaling method may be expressed by its ability to deduce the inviscid propeller (no surface friction acting) from any Reynolds number level. To quantify a Reynolds number level a unique Reynolds number may be defined by $\operatorname{Re}_{0.7}$.

$$
\operatorname{Re}_{0.7}=\frac{\mathrm{c}_{0.7}}{v} \sqrt{\omega^{2} \mathrm{r}_{0.7}^{2}+\mathrm{u}^{2}}
$$

where:

$\omega-$ angular frequency,

$\mathrm{u}-$ axial inflow velocity,

$\mathrm{r}_{0.7}-70 \%$ of propeller radius,

$\mathrm{c}_{0.7}-$ chord at $\mathrm{r}_{0.7}$,

$v-$ kinematic viscosity).

The bigger problem for scaling is considered to occur at model scale level, but it can hardly be proved whether a method performs well in this range or not. The problem with ranking scaling methods is related to the fact that the inviscid propeller is hard to access experimentally and numerically.

Using a strip method gives at least a chance to resolve the mixture of laminar and turbulent flow at any radial position. As a basic characteristic of friction on a propeller blade a Reynolds number range is to be considered (instead of relying on one unique Reynolds Number like $\operatorname{Re}_{0.7}$ ). Basically the Reynolds number changes strongly along the radius due to the local rotational speed. The strip method accounts for this situation. Furthermore, it accounts for the blade outline as well as for the blade pitch distribution and sums up friction effects from the set of strips that built up the blade.

This principle is displayed in Fig. 2, showing a coarse arrangement of strips and a color code to indicate the significance of each strip. Each strip has its local pitch and converts section drag into a local mixture of axial and circumferential force components. Finally, the latter shares are summed up to arrive at a thrust coefficient correction $\Delta \mathrm{K}_{\mathrm{T}}$ and a torque coefficient adjustment $\Delta \mathrm{K}_{\mathrm{Q}}$ for the whole propeller.

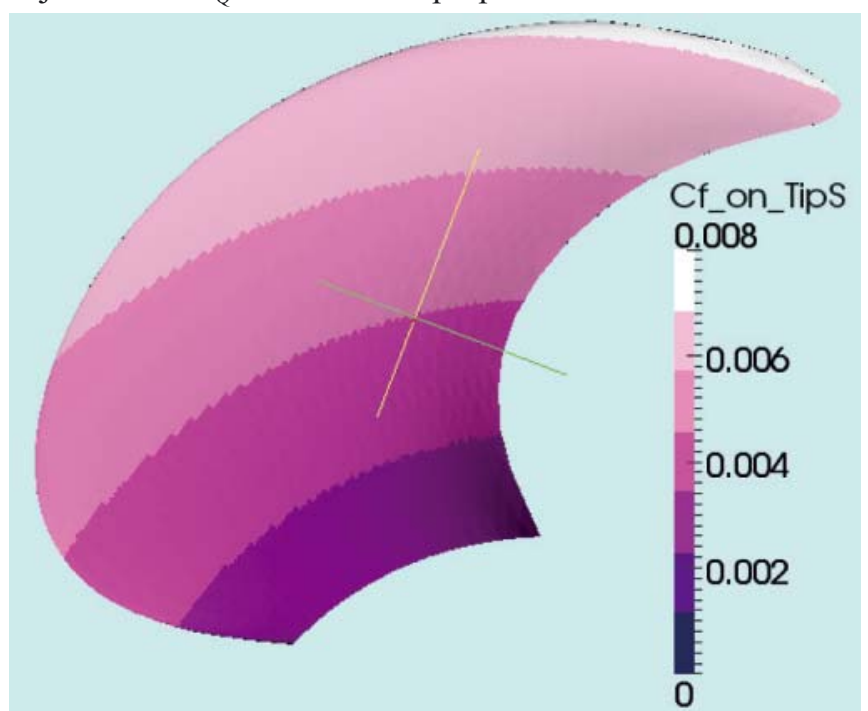

Fig. 2. Principle of the strip method representing the relevance of different strips for the total viscosity effect (significance increases with brightness)

\section{Concept of the new HSVA procedure based on the strip method}

In a review of HSVA's former standard procedure (the Lerbs/Meyne-method) the following improvements, together with the introduction of a strip method, were considered:

- address full scale in a more definite manner compared to the standard methods (Lerbs/Meyne-method [5] formerly used at HSVA, or ITTC'78-method [4]), 
- withdraw the charts-based process to identify an 'ideal' propeller for further interpretation of the open water test and for full scale performance prediction (an essential idea behind the Lerbs/Meyne-method to recognize the above mentioned inviscid propeller),

- instead, rely on a more conventional scaling process, i.e. use friction lines to arrive at all scales starting with the measured open-water performance,

- introduce different friction lines for the open-water test mode and the propulsion test mode to resolve for different levels of incoming turbulence in either case.

The idea to use two friction lines for either the open water mode or the propulsion mode shall reflect different characters of the incoming flow. The lines are related to different degrees of turbulence in the arriving flow affecting the mixture of laminar and turbulent regimes on the blade surface. Fig. 3 gives the basic friction lines representing section drag in a normalized form, which is to be linked to the drag D of a strip (supposed to act in the direction of the nose-tail line) by means of:

where:

c - represents the chord of the strip,

$\mathrm{dr}$ - its radial extension

$\mathrm{V}_{0}$ - the relevant velocity, the latter including rotation and advance of the strip.

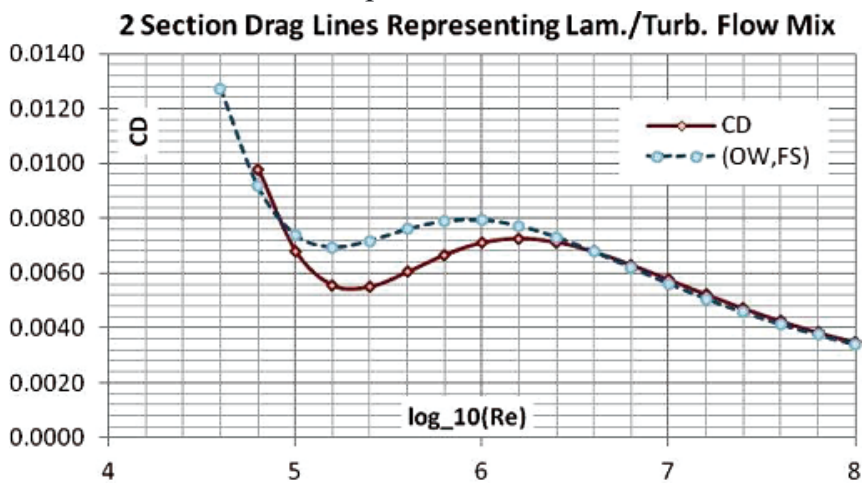

Fig. 3. Lines for section drag coefficient used in the new strip method: the full red line stands for the full scale and open water case (OW, FS), the dashed blue line - for the propulsion test mode (RP). Each line reflects a specific mixture of laminar and turbulent flow.

\section{Validation and correlation of the new HSVA procedure}

The inviscid propeller concept is detailed in Fig. 4. One might avoid this idea and reduce the process of scaling to finding the difference between friction forces in model scale and in full scale. This view may however suggest certain simplicity and it is recommended here to retain the "back and forth"- view as given in Fig. 4.

For an experimental based access to the inviscid propeller it is considered reasonable to reduce mixture effect from simultaneous appearance of laminar and turbulent flow as far as possible. In the experiments it was aimed at the largest Reynolds numbers achievable with existing propeller hardware and test facilities. It was expected that by doing so the above mentioned mixtures effects were reduced and the prospects for ranking scaling procedures were enhanced. At HSVA the large model propellers were tested in the largest conventional cavitation tunnel (where the measuring equipment can stand higher forces and moments as the towing tank hardware). Ensuring that turbulent flow becomes dominant on the blade surface it was considered that empirical lines for pure turbulent blade surface friction can be used to arrive at a good estimation of viscous forces.

Evaluating these tests it was soon noticed that the standard ITTC'78 method and the 'Lerbs/Meyne'- method previously applied at HSVA, may treat full scale friction effects too conservatively. For the ITTC' 78 method this is obvious from Fig. 4, where for propeller 2004 the full scale $\eta$ was predicted slightly below model scale efficiency (using the standard roughness setting). This finding rather supports the necessity of a detailed treatment of viscous effect in the range of full scale Reynolds numbers (which was not addressed in the Lerbs/Meyne-method). It gives no further arguments for the necessity of a strip method.

Besides the propeller efficiency scaling process, the evaluation of propulsion test results for a full scale power prediction involves other correction steps, among which are wake-scaling and added resistance. It was an essential demand for the modification of propeller efficiency scaling, that it should not lead to a general shift of predicted full scale power (in the mean sense). This led to the necessity to run a lot of comparative evaluations of propulsion tests results in both ways, applying the previous process and the new strip method.

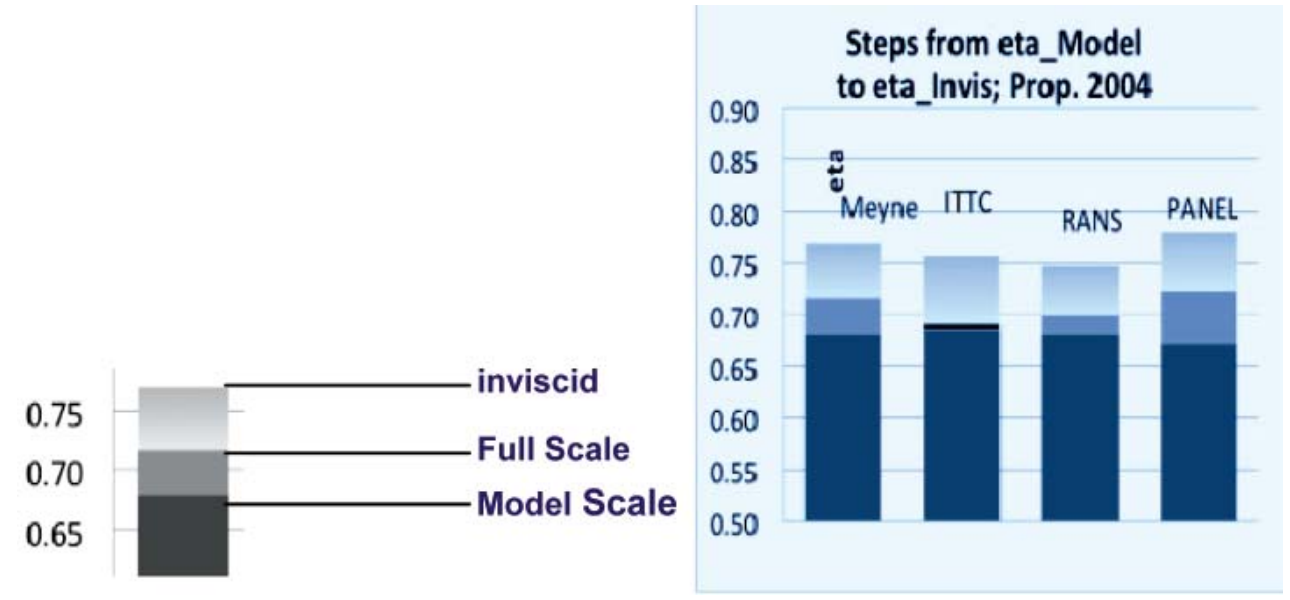

Fig. 4. Illustration of the inviscid propeller concept (left). The model scale efficiency is measured, the estimation and subtraction of model scale surface friction forces gives the in-viscid performance and a guess for full scale friction effects reduces the inviscid efficiency to the full scale efficiency. The diagram on the right holds for propeller $2004(\mathrm{D}=0.382 \mathrm{~m})$, operated at $18 \mathrm{~Hz}\left(\mathrm{Re}_{0.7}=2.3 * 10\right)$ in HSVA's large conventional tunnel. Various methods were used to execute the steps explained on the left. For propeller 2004 the full scale efficiency level in the ITTC column (reflecting the application of the ITTC'78 scaling procedure) is hardly visible as it was predicted slightly below model scale efficiency. 
For a particular propeller Fig. 5 gives a comparison of either method when applied over the whole range of advance ratios $\mathrm{J}$. The focus is on the efficiency $\eta=\mathrm{J} \cdot \mathrm{K}_{\mathrm{T}} / 2 \pi \mathrm{K}_{\mathrm{Q}}$, where in the figure $\eta_{\text {OPEN WATER }}$ stands for the unaltered measured quantity; $\eta_{\mathrm{FS}}$ and $\eta_{\text {PROPULSION }}$ are related to full scale and propulsion test, respectively. It may be considered a drawback of the previous approach, that in some particular cases (the given example belongs to this set) the steps between the 3 relevant efficiencies seem to be of reversed order. The strip method predicts the propulsion mode efficiency closer to $\eta_{\text {OPEN WATER }}$ and further from $\eta_{\mathrm{FS}}$ and not opposite, as the former approach does.
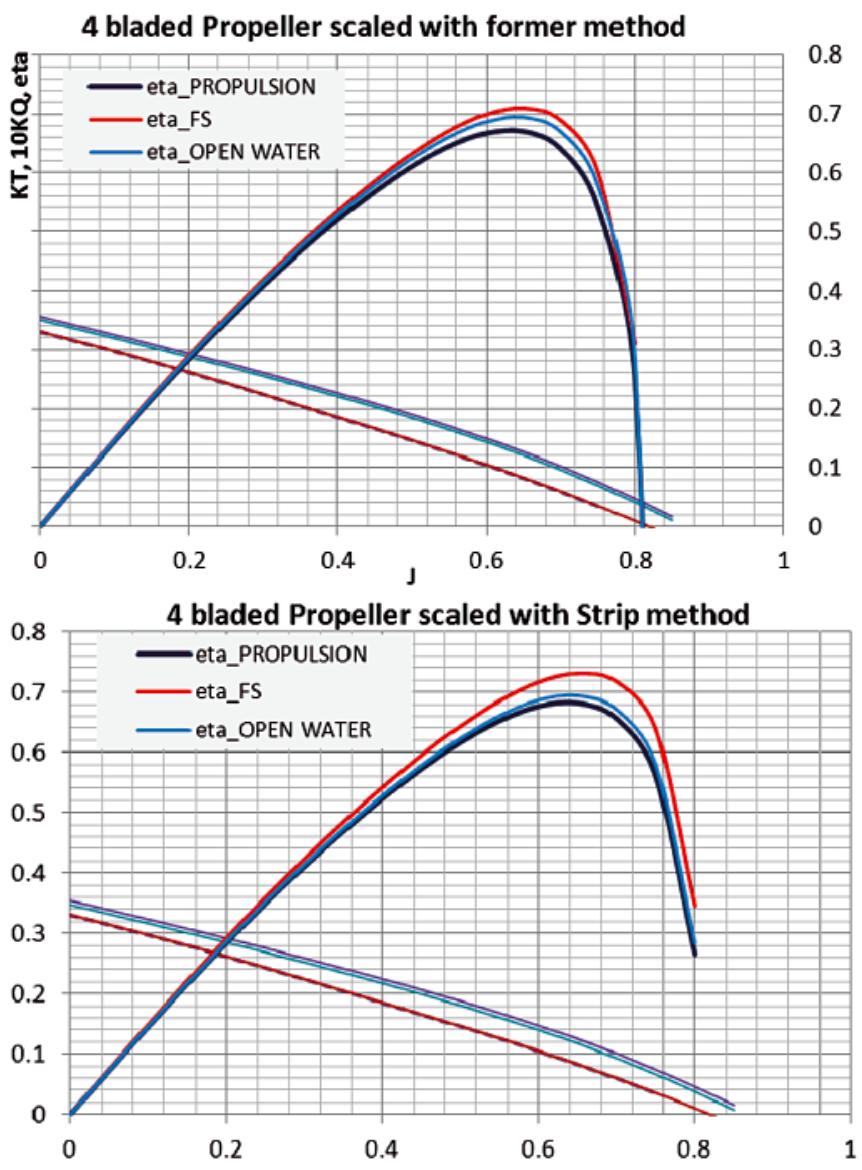

Fig. 5. Full scale efficiency versus propulsion test efficiency for 4-blade propeller obtained from the former HSVA scaling procedure and due to the new strip method

$\mathrm{J}=0.6$, Propeller 2188

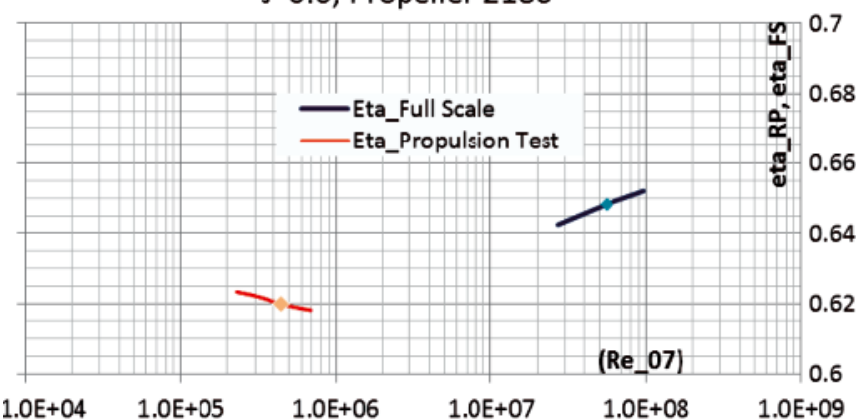

Fig. 6. The open-water efficiency plotted for a specific advance ratio over the Reynolds number on $70 \%$ of the propeller radius, considering ranges

typical for full scale or model scale (propulsion mode) conditions

Fig. 6 depicts one advance ratio and shows the variation of the open water efficiency with the Reynolds Number at $70 \%$ of the propeller radius. A typical full scale range and a typical model scale range (for a propulsion mode) are considered. This figure reflects the character of the above given section drag lines. Note that when using a separate friction line (the
RP-line) the efficiency for propulsion conditions is generally worsened. The RP-line however shows a local minimum for typical propulsion test Reynolds numbers.

It was considered reasonable to post-process RANS results in a manner that section drag can be presented strip-wise to allow for a direct comparison with the strip method. The procedure is demonstrated in Fig. 7. The integration of forces generated by shear is confined to a strip and local velocities and the strip area are taken for reference to calculate a section drag coefficient by using the above given $C_{D}$ - formula. For the same propeller the $C_{D}$ values obtained from the strip method approach may be collected.

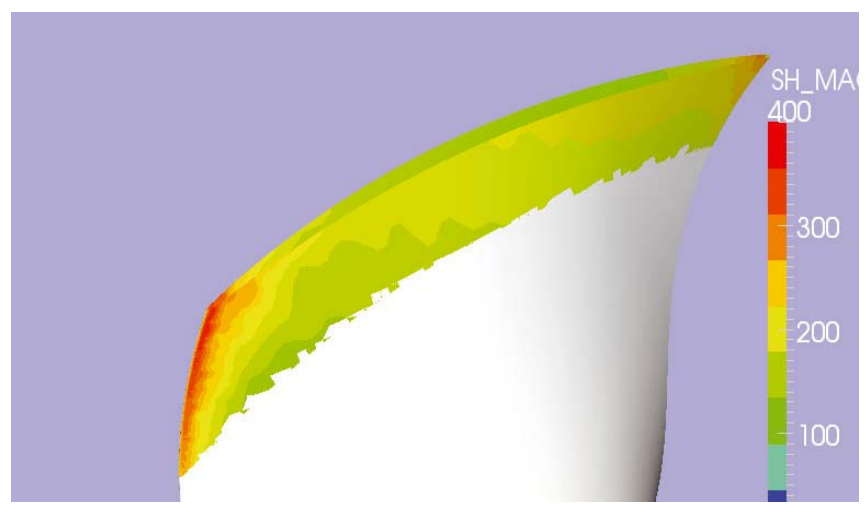

Fig. 7. Shear force distributions from RANS results, used to derive a strip - wise resolution of section drag (due to shear) for comparison with strip method results

Fig. 8 gives $\mathrm{C}_{\mathrm{d}}$ results plotted against the radius, referencing RANS calculations performed with the HSVA's in-house solver FreSCo and strip method assumptions by using the above given section drag lines. As the RANS solver was assuming turbulent flow starting from the leading edge it was expected that it would reveal a different behaviour over the radius. The relation of measured shaft power from trials to predicted shaft power from propulsion tests should improve with the new correction method, though (in the mean sense) the predictions should not deviate too much from the HSVA's former procedure.

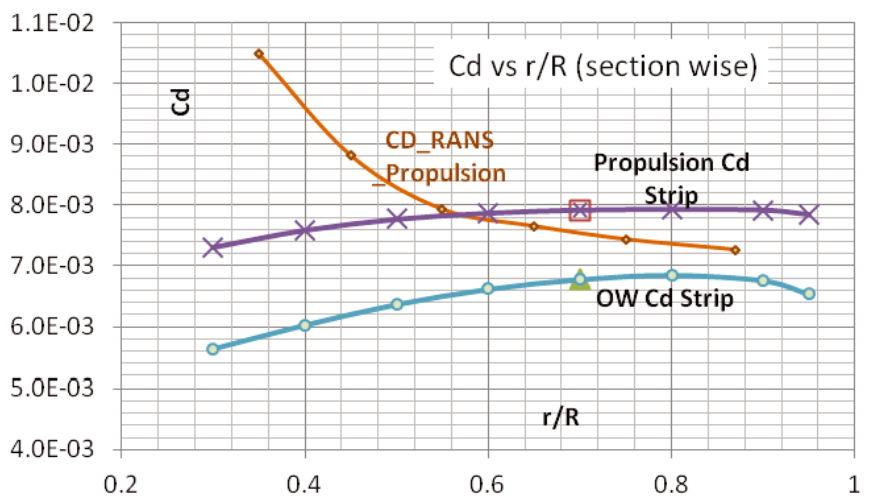

Fig. 8. Instead of plotting section drag vs. Reynolds number one may indirectly consider the section drag coefficient in function of the radius

Fig. 9 (up) gives all 112 trial cases available at HSVA, re-evaluated with the new method from model tests and then compared with the actual measured power. Fig. 9 (down) displays the former correlation obtained with the Lerbs/Meyne approach. Comparing both figures one can state that the application of the strip method improved the correlation (1.6\% under this prediction vs. $2.5 \%$ under prediction of power) but the results stayed only $0.9 \%$ apart, as both procedures tend to under-predict. 

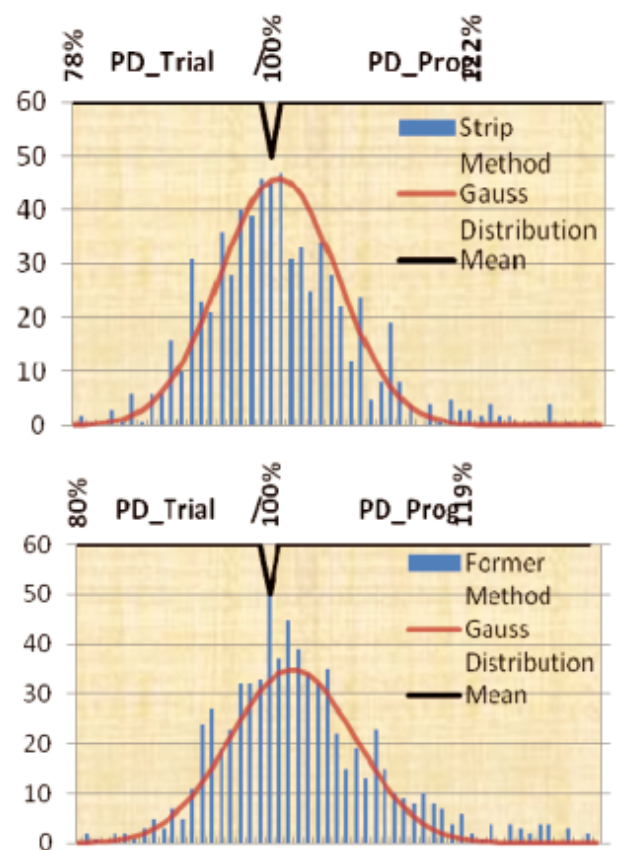

Fig. 9. Comparison of the strip method (up) and the former method (down) in terms of their ability to match $100 \%$ correlation in average. The mark $(\nabla)$ indicates the position for a case where $100 \%$ match of predicted and measured power was achieved $\left(P D_{\text {Trial }}=P D_{P r o g}\right)$. The actual mean for the ratio trial-power vs. predicted power $\left(P D_{\text {Trial }} / P D_{\text {Prog }}\right)$ is to be taken from the maximum of the Gauss-distribution

\section{RESEARCH AT THE SHIP DESIGN AND RESEARCH CENTRE (CTO SA)}

\section{Alternative formulae for lift and drag of propeller blade sections}

The currently used ITTC78 procedure [4] for development of scale effect corrections for propeller thrust and torque is often criticized in three aspects:

- the formulae for propeller scale effect are based on the equivalent blade section at 0.75 radius, therefore they may be inadequate for contemporary propeller geometries, especially these with high skewback and strongly nonuniform radial pitch distributions,

- the formulae used for calculation of blade section drag may be not accurate enough,

- the scale effect on blade section lift may have to be included ([8]).
The alternative formula for blade section drag coefficient, in model and full scale alike, considered in this paper in Variants 3 and 4 below, has the following form (taken from [1]):

$$
C_{D}=0.05808\left(1-0.2 \frac{t}{c}\right) \frac{1}{\operatorname{Re}^{0.1458}}
$$

The formula for scale effect on blade section lift coefficient considered in this paper in Variant 3 below, has the following form (also taken from [1]):

$$
\mathrm{C}_{\mathrm{LV}}=2 \pi \mu_{1}\left(\alpha+\mu_{2} \alpha_{0}\right)
$$

where:

$$
\begin{gathered}
\mu_{1}=1-\exp \left(-0.0691+12.46 \frac{\mathrm{t}}{\mathrm{c}}-0.1855 \log R \mathrm{e}\right) \\
\mu_{2}=1+\frac{\mathrm{t}}{\mathrm{c}}\left(\frac{\mathrm{t}}{\mathrm{c}}-0.05\right) \frac{1}{(0.04664 \log \operatorname{Re}-0.4378)^{2}} \\
\operatorname{Re}=\frac{\mathrm{c}}{v} \sqrt{\omega^{2} \mathrm{r}^{2}+\mathrm{u}^{2}}
\end{gathered}
$$

\section{Model propellers used in the experiments and calculations}

Altogether three propeller models were analyzed at CTO SA: one of them (P629) has a more traditional geometry, resembling the $\mathrm{B}$ Wageningen - series propellers, and the other two are highly skewed propellers CP312 and CP416. Only the results for highly skewed propellers are presented in this article. The geometry of the propeller CP312 is shown in Fig. 10, and the geometry of propeller CP416 is presented in Fig. 11. The geometry and results for the propeller P629 may be found in [3].

\section{Towing tank model experiments at CTO SA}

The open water experiments with the above presented propeller models were conducted in the large towing tank of CTO SA. The standard open-water diagrams of both propeller models were measured. Apart from that, the influence of Reynolds number on the propeller hydrodynamic characteristics was measured for the given advance coefficients by changing the rotational speed of the models within the range permitted by the available measuring equipment and the maximum possible advance velocity of the towing tank carriage. The Reynolds
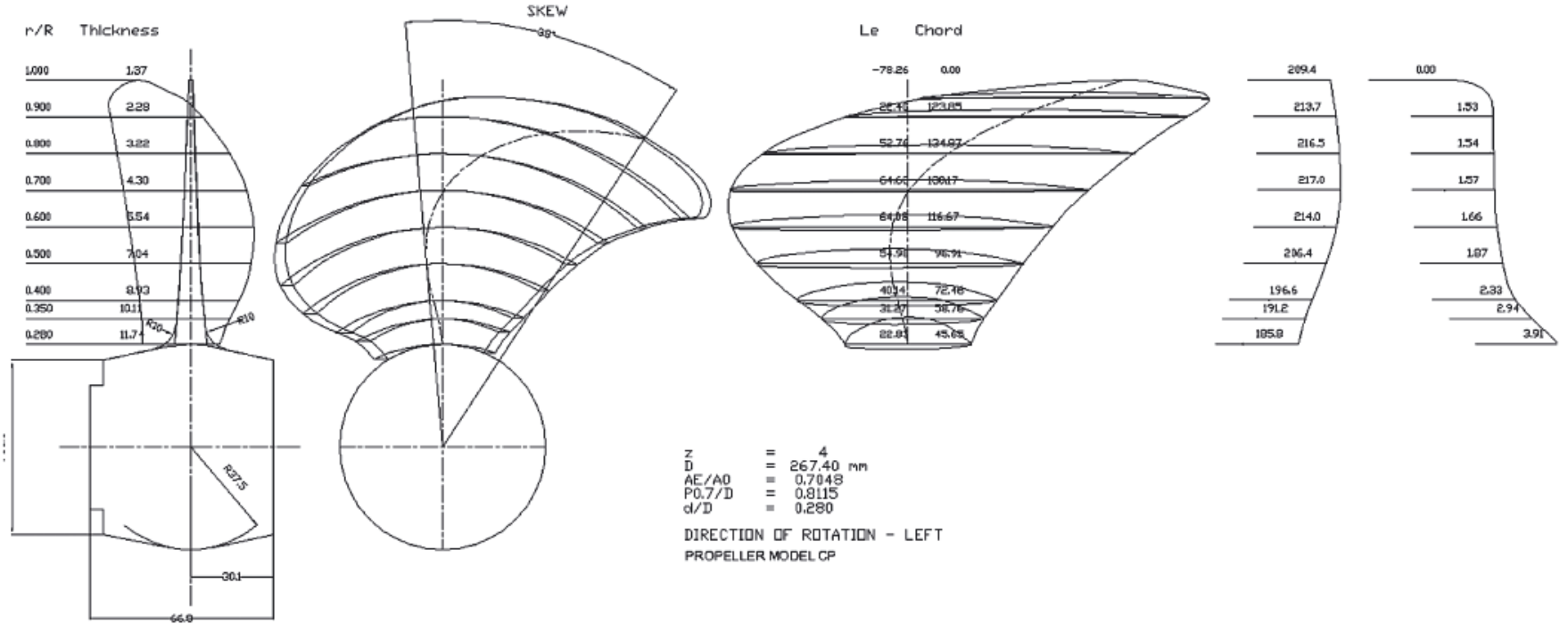

Fig. 10. Model propeller CP312 tested and analyzed at CTO SA 


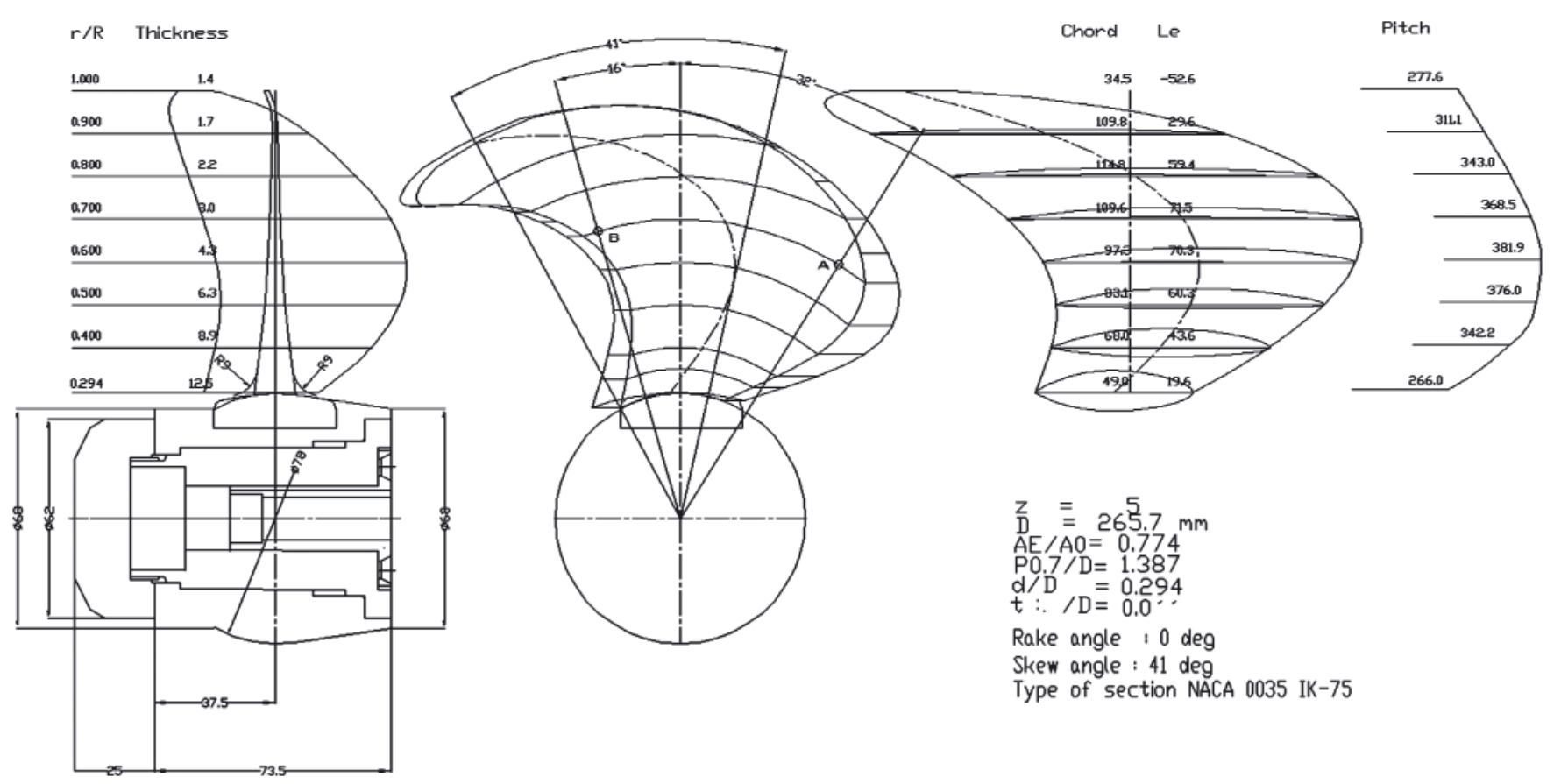

Fig. 11. Model propeller CP416 tested and analyzed at CTO SA

Tab. 1. Reynolds numbers in tank tests and in calculations at CTO SA (all values *106)

\begin{tabular}{|c|c|c|c|c|c|c|c|}
\hline \multicolumn{8}{|c|}{ Open-water tests in towing tank } \\
\hline Propeller & $\mathrm{J}$ & $\mathrm{n}=4[1 / \mathrm{s}]$ & $\mathrm{n}=6[1 / \mathrm{s}]$ & $\mathrm{n}=8[1 / \mathrm{s}]$ & $\mathrm{n}=10[1 / \mathrm{s}]$ & $\mathrm{n}=12[1 / \mathrm{s}]$ & $\mathrm{n}=16[1 / \mathrm{s}]$ \\
\hline \multirow[t]{2}{*}{$\mathrm{CP} 312$} & 0.50 & - & - & 0.573 & 0.707 & 0.852 & 1.135 \\
\hline & & $\mathrm{n}=4[1 / \mathrm{s}]$ & $\mathrm{n}=6[1 / \mathrm{s}]$ & $\mathrm{n}=8[1 / \mathrm{s}]$ & $\mathrm{n}=10[1 / \mathrm{s}]$ & $\mathrm{n}=12[1 / \mathrm{s}]$ & $\mathrm{n}=16[1 / \mathrm{s}]$ \\
\hline CP416 & 0.70 & 0.225 & 0.338 & 0.450 & 0.563 & 0.676 & - \\
\hline \multicolumn{8}{|c|}{ CFD calculations for model scale } \\
\hline & & $\mathrm{n}=13[1 / \mathrm{s}]$ & $\mathrm{n}=20[1 / \mathrm{s}]$ & $\mathrm{n}=30[1 / \mathrm{s}]$ & $\mathrm{n}=40[1 / \mathrm{s}]$ & $\mathrm{n}=50[1 / \mathrm{s}]$ & $\mathrm{n}=60[1 / \mathrm{s}]$ \\
\hline $\mathrm{CP} 312$ & 0.50 & 0.973 & 1.497 & 2.245 & 2.984 & 3.742 & 4.491 \\
\hline $\mathrm{CP} 416$ & 0.90 & 0.862 & 1.326 & 1.988 & 2.651 & 3.314 & 3.977 \\
\hline
\end{tabular}

numbers achieved in experiments for both propeller models are given in Tab. 1 together with the numbers used in calculations.

The results of towing tank experiments are presented in Fig. 12 and 13. They confirm the expected tendencies of all coefficients, but they do not reach the Reynolds numbers considered in calculations below. Therefore they cannot be used for direct validation of the results of different variants of calculation presented below in Fig. 16, 17, 18 and 19.

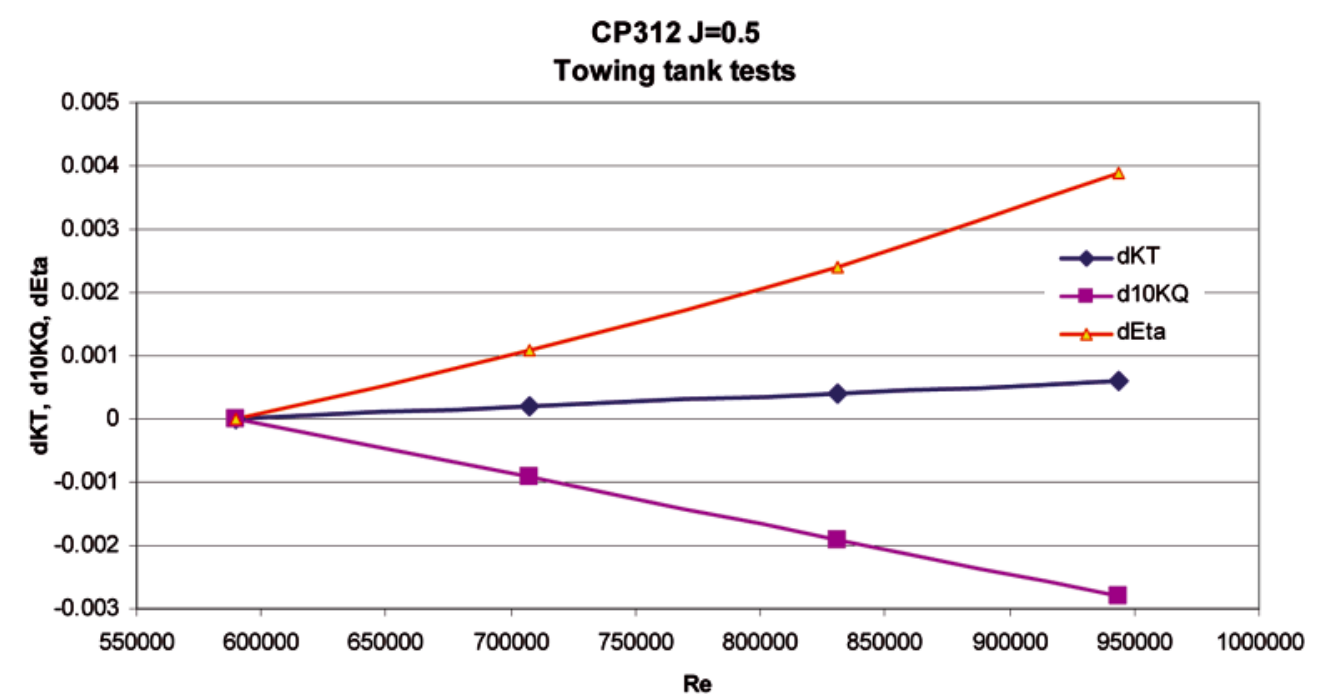

Fig. 12. Scale effect corrections for the thrust coefficient, torque coefficient and efficiency of the propeller model CP312 based on measurements in the towing tank at different Reynolds numbers 
CP416 J=0.6

Towing tank tests

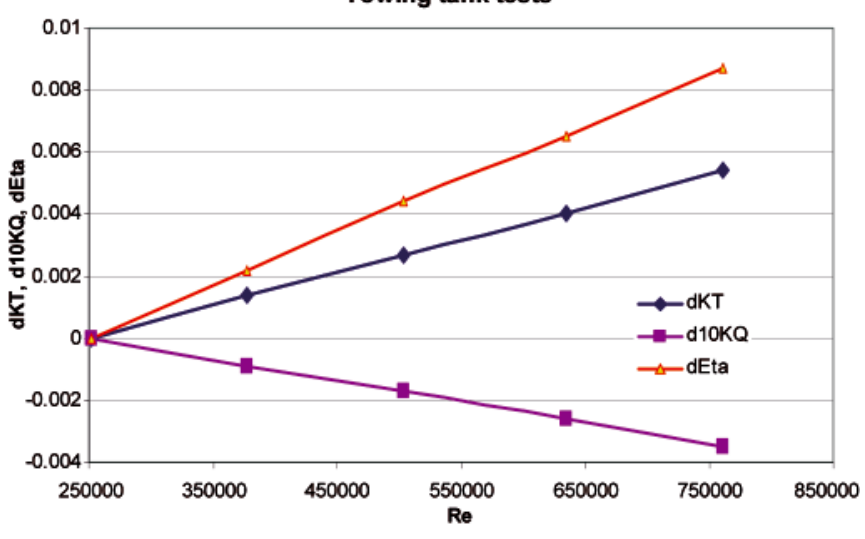

Fig. 13. Scale effect corrections for the thrust coefficient, torque coefficient and efficiency of the propeller model CP 416 based on measurements in the towing tank at different Reynolds numbers

\section{Lifting surface and CFD calculations at the Ship Design and Research Centre (CTO SA)}

The well-established and thoroughly validated lifting surface program UPCA92 for analysis of propeller operation in non-uniform velocity field [9], was used. The program was modified in such a way that it produced the complete open-water diagram of propellers at the required scale. In the calculations the propeller rate of rotation was usually kept constant and the advance velocity was varied in order to produce the required values of the advance coefficient. Four different algorithms for prediction of scale effect corrections were incorporated into the program:

- the classical ITTC78 procedure (hereinafter Variant 1), based on the equivalent blade section at radius 0.75

- the ITTC78 formulae used locally for the respective blade sections and whose results are integrated along the blade radius - similarly as in the above described HSVA's strip method (hereinafter Variant 2),

- alternative formulae for blade section drag and lift integrated along the blade radius (hereinafter Variant 3),

- alternative formula for blade section drag only integrated along the blade radius (hereinafter Variant 4).

The program was used for calculations in model scale for $13,20,30,40,50$ and $60 \mathrm{rps}$ and for calculation of three different simulated full-scale situations, corresponding to propeller diameters of 3,5 and $8[\mathrm{~m}]$. The full-scale calculations were performed for standard propeller blade roughness value of 30 microns.

Tab. 2. Reynolds numbers in simulated full-scale calculations (all values $* 10^{8}$ )

\begin{tabular}{|c|c|c|c|}
\hline Propeller & $\mathbf{D}=\mathbf{3 . 0}[\mathbf{m}]$ & $\mathbf{D}=\mathbf{5 . 0}[\mathbf{m}]$ & $\mathbf{D}=\mathbf{8 . 0}[\mathbf{m}]$ \\
\hline CP312 & 0.3768 & 0.6781 & 1.050 \\
\hline CP416 & 0.3378 & 0.5633 & 0.9012 \\
\hline
\end{tabular}

In the CFD calculations the meshing and flow simulations are performed with the Reynolds Averaged Navier-Stokes (RANS) solver StarCCM+ from CD-Adapco. The code solves the RANS and continuity equations in integral form on a polyhedral mesh by means of the finite volume technique. The Reynolds stress problem is solved by means of "realizable" $k-\varepsilon$ turbulence model. As in the open-water situation the propeller inflow is uniform the moving reference frame approach is applied. The solution domain was chosen such as to extend 10 propeller diameters in front of the propeller, 3 diameters in the radial direction and 5 diameters behind the propeller. The computational grid was constructed of about 900000 polyhedral elements. The details of the calculation technique may be found in [2].

The CFD calculations were carried out for advance coefficients in the range from $\mathrm{J}=0.0$ to $\mathrm{J}=1.0$, following the above defined model and full-scale conditions for the lifting surface calculations. The computed thrust and torque on the propeller were converted into the dimensionless thrust coefficient, torque coefficient, and the propeller open-water efficiency was calculated. The study of the flow field shows that the phenomena occurring in the flow (pressure distribution, tip vortices etc) are typical for propeller flow and can be considered qualitatively correct.

In Fig. 14 is shown the calculated distribution of the local viscous shear stress and pressure distribution on the suction side of the model propeller CP416. The distribution of shear stress supports the idea of dividing the blade surface into strips and calculating friction forces separately for each strip. The analogous results for full scale presented in Fig. 15 do not support the idea of dividing the blade surface into strips in an equally clear way. However, it is visible that the shear stresses are distributed over the blade surface in a strongly non-uniform way and describing them with a single drag coefficient is a very far reaching approximation.

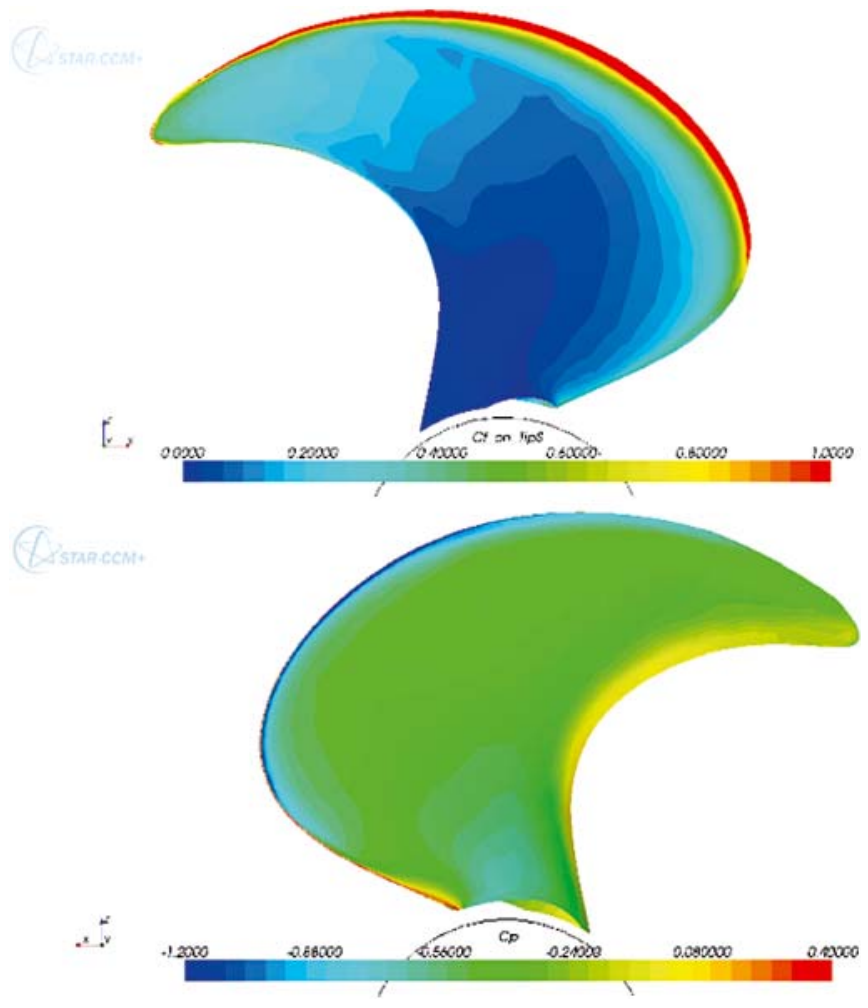

Fig. 14. CFD calculations for propeller model CP416: blade surface shear stress distribution based on tip speed (up) and pressure distribution on the suction side (down)

\section{Results of computations obtained at the Ship Design and Research Centre (CTOSA)}

The results of computations for the propeller CP312 are shown in Fig. 16 and 17 in the form of scale effect corrections related to the model scale values of respective parameters at the lowest Reynolds number, i.e. at 13 rps. The scale effect corrections are shown for the design advance coefficient of the propeller equal to $\mathrm{J}=0.5$. Fig. 16 shows the corrections for 


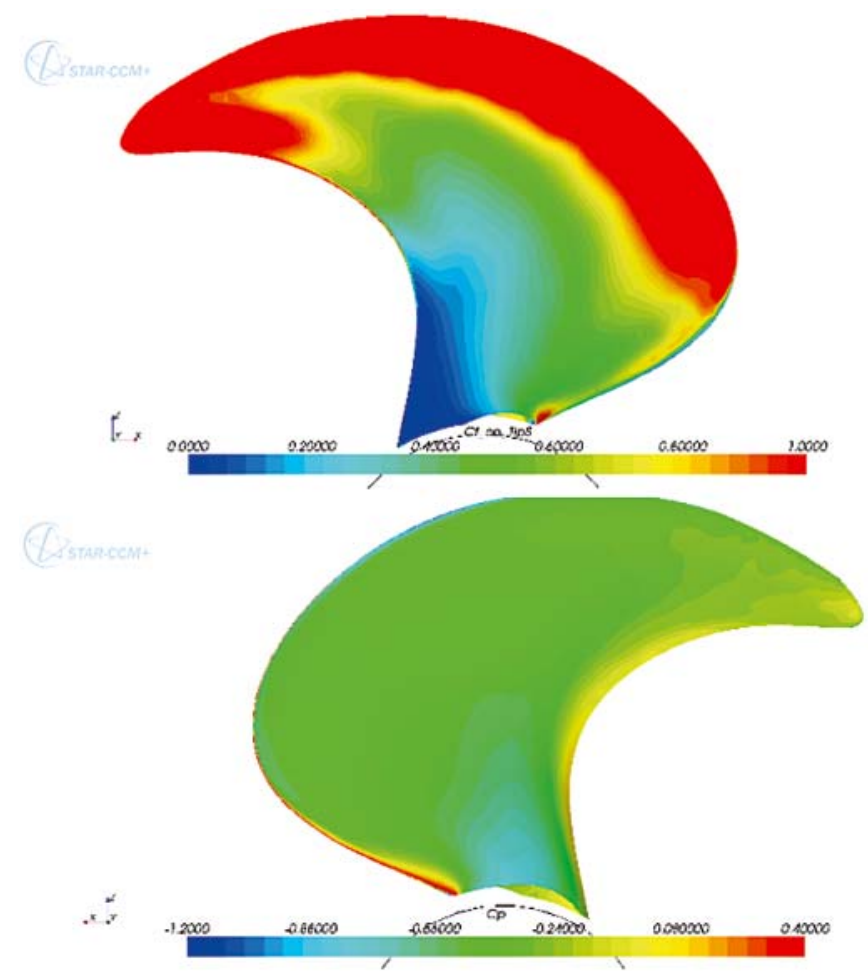

Fig. 15. CFD calculations for full scale $(D=5 \mathrm{~m})$ propeller CP416: blade surface shear stress distribution based on tip speed (up) and pressure distribution on the suction side (down)

propeller torque coefficient and Fig. 17 - for propeller openwater efficiency.

The scope of the calculations and the form of presentation of the results for propeller CP416 in Fig. 18 and 19 are identical to those for propeller CP312. The design advance coefficient is now $\mathrm{J}=0.9$.

The above presented results of lifting surface and CFD calculations may be summarized in the following way, assuming CFD results as the level of reference:
- the classical ITTC78 formulae produce too high corrections for propeller torque, effectively under-estimating this parameter in full scale; this may lead to the development of hydrodynamically "too heavy" propeller designs;

- the classical ITTC78 procedure significantly over-estimates the increase in propeller open-water efficiency in full scale as compared with model scale; this may cause too optimistic predictions of ship powering performance;

- the integration of the locally defined ITTC78 corrections along the propeller radius does not produce visible changes in the results when the presence of laminar/turbulent flow in model scale is not taken into account;

- variant 3 based on the alternative formulae for blade section lift and drag produces too high corrections for propeller thrust, but comparison with ITTC78 - based results shows that some smaller scale effect on lift may need to be included in the scaling procedure in order to improve correlation of the thrust correction with CFD results,

- the alternative formula for blade section drag, used in Variants 3 and 4, seems to predict scale effect corrections for torque much better than the original ITTC78 formula.

\section{GENERAL CONCLUSION}

The research performed both at HSVA and CTO SA leads to the following general conclusions:

- the currently used procedures for propeller scale effect corrections require modifications in order to keep up with the advanced propeller designs and advanced experimental and numerical techniques;

- to develop the reliable new procedures is difficult due to the complicated nature of the involved physical phenomena;

- the incorporation of the new formulae for blade section drag coefficients, taking into account the mixed laminar/turbulent flow in model scale, is a promising concept which requires further investigation;

- the incorporation of the scale effect on blade section lift may be also the way to improve the current scale effect procedures.

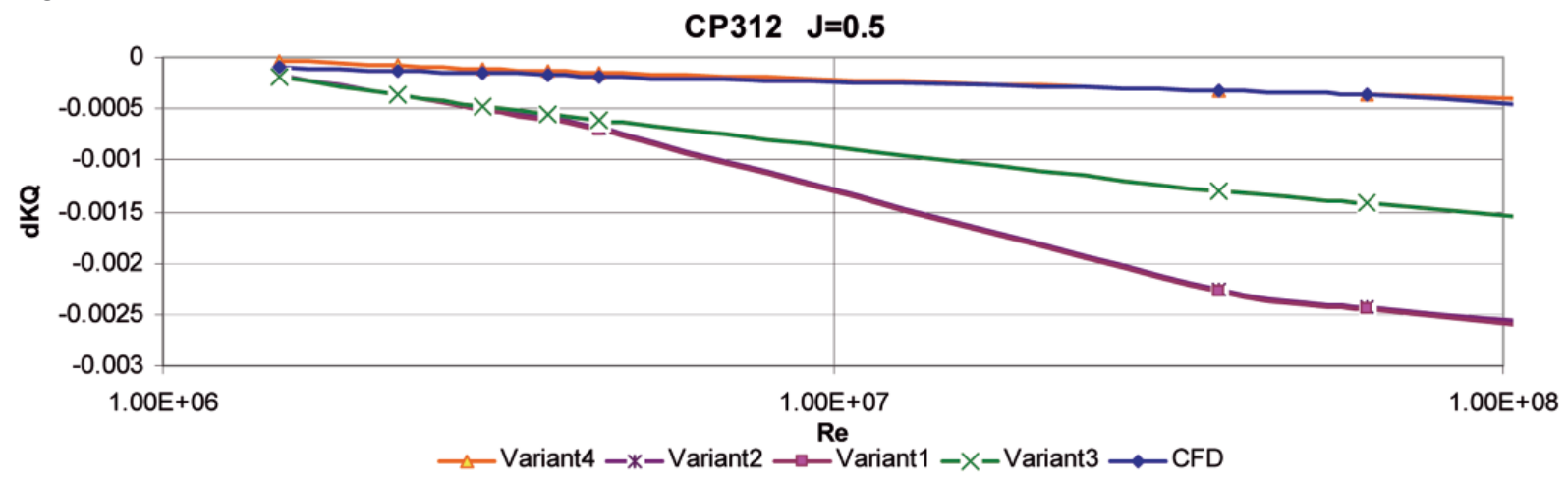

Fig. 16. Results of calculations for the scale effect correction on the torque coefficient of the CP312 propeller

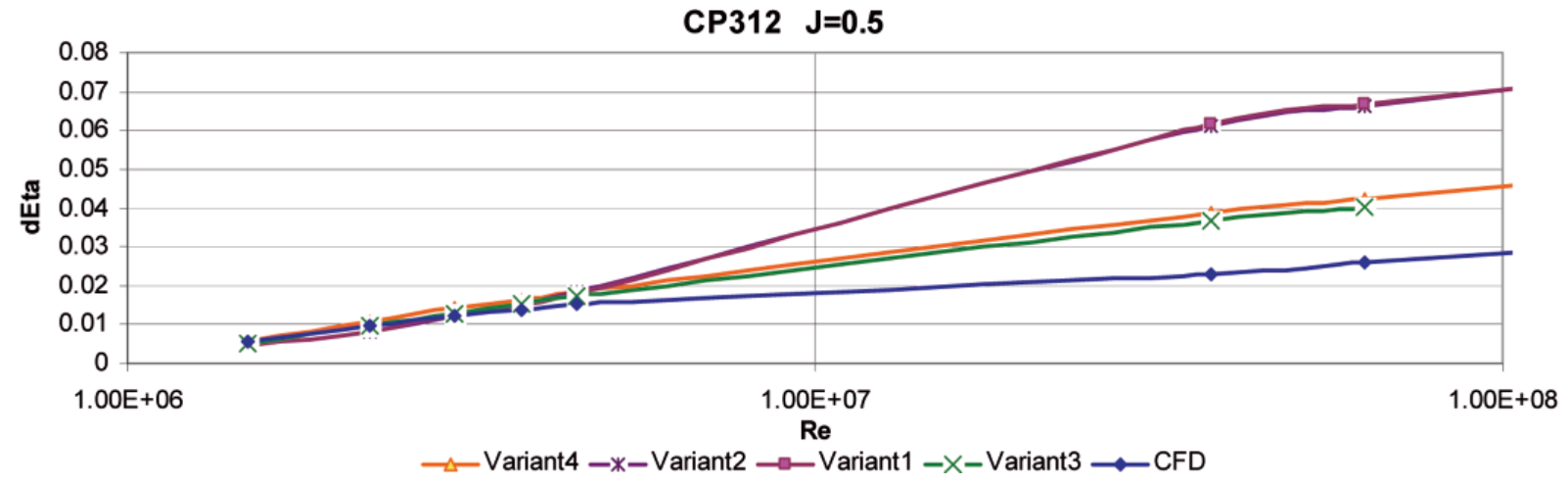

Fig. 17. Results of calculations for the scale effect correction on the open-water efficiency of the CP312 propeller 


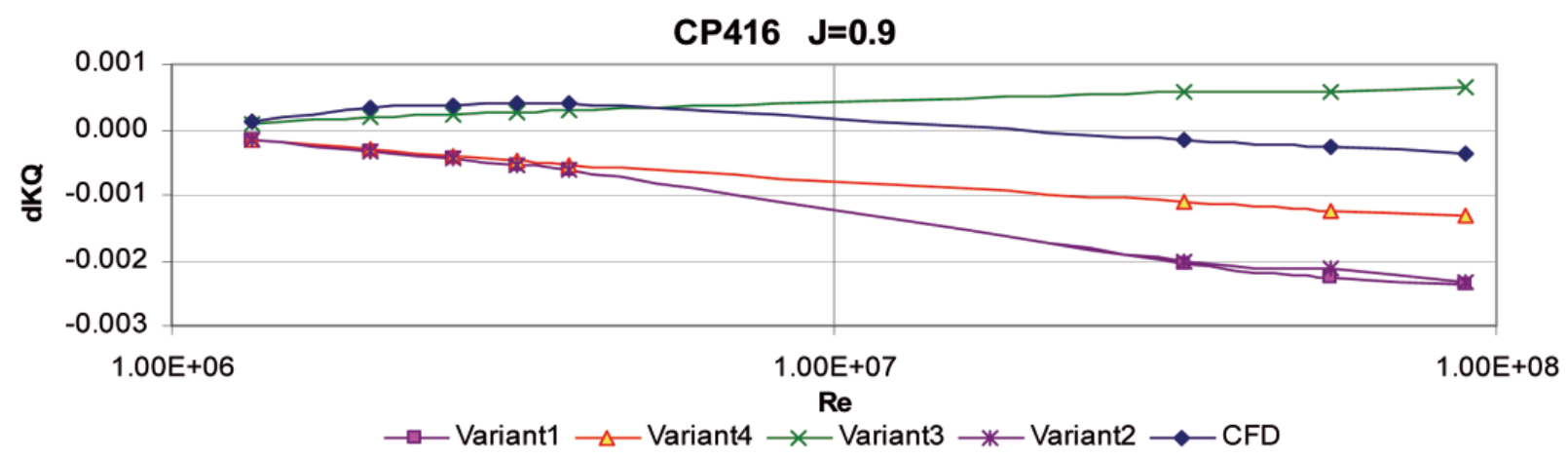

Fig. 18. Results of calculations for the scale effect correction on the torque coefficient of the CP416 propeller $(J=0.9)$

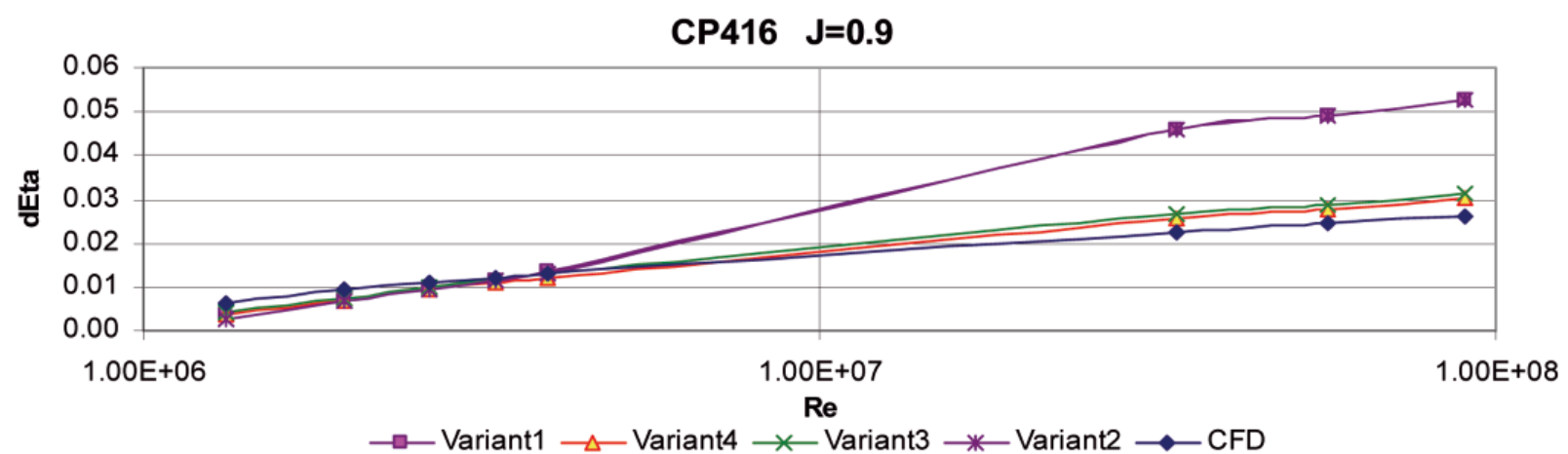

Fig. 19. Results of calculations for the scale effect correction on the open-water efficiency of the CP416 propeller $(J=0.9)$

\section{Acknowledgements}

The authors acknowledge with gratitude that the research presented in this paper was supported by the ERA-NET MARTEC and by the Polish National Centre for Research and Development (NCBiR) grant No. N R10 0039 06/2009.

\section{NOMENCLATURE}

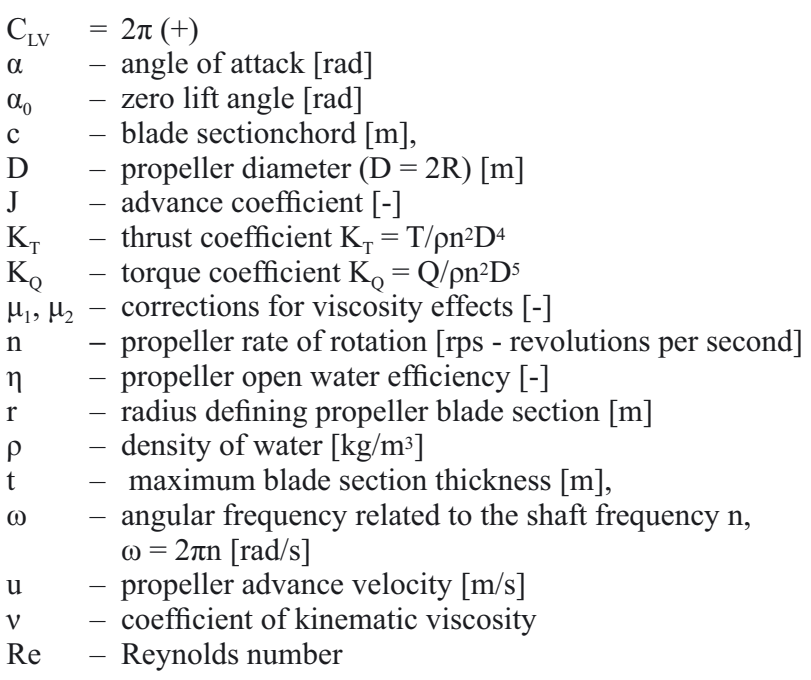

\section{BIBLIOGRAPHY}

1. Bavin W.F. et al: Grebnye winty -sovremennyje mietody razcziota (in Russian), Izd. Sudostrojenije, Leningrad 1983

2. Bugalski, T., Hoffmann, P.: Numerical Simulation of the Interaction Between Ship Hull and Rotating Propeller.

Proceedings of the Second International Symposium on Marine Propulsors, SMP'11, Hamburg, Germany, 2011, pp. 256-26

3. Bugalski T., Szantyr J.A.: Numerical Analysis of Scale Effects in Open Propellers. Proc. International Symposium on Ship Hydrodynamics HYDRONAV 2012, Ilawa, September 2012

4. ITTC Performance Committee: Report of the Performance Committee. Proceedings of the $15^{\text {th }}$ ITTC, 1978
5. Meyne, K.: Experimentelle und theoretische Betrachungen zum Maßstabseffekt bei Modellpropeller-Untersuchungen (in German) Schiffstechnik, Vol. 15, 1968.

6. PREFUL - Propeller Efficiency in Full Scale. European research project under the MARTEC network, $2010 \div 2012$

7. Streckwall H., Dymarski P., Greitsch L.: Review of propeller performance scaling including RANS calculations and high speed tunnel tests. HydroComp 2012, Propeller Workshop, Glasgow, 11 June, 2012.

8. Szantyr J.: Lift Based Propeller Scale Effect and its Influence on the Propulsive Characteristics of Ships, Trans. West Japan Society of Naval Architects, No. 84 (1992), pp. 239-246

9. Szantyr J., Centkowski J.: UPCA92 - the Lifting Surface Program for Hydrodynamic Analysis of Marine Propellers. Part II-Description of the Algorithm, (in Polish). Report of IFFM ? No. 22/92

\section{CONTACT WITH THE AUTHORS}

Tomasz Bugalski Ph.D.,

Ship Design and Research Centre CTO SA, Szczecinska 65,

80-392 Gdansk, POLAND, e-mail: tomasz.bugalski@cto.gda.pl

Heinrich Streckwall, Ph.D., Hamburg Ship Model Basin (Hamburgische Schiffbau-Versuchsanstalt GmbH), Bramfelder Str. 164,

D-22305 Hamburg, GERMANY, e-mail: Streckwall@hsva.de

Jan A. Szantyr, Prof., Faculty of Mechanical Engineering, Gdansk University of Technology, Narutowicza 11/12, 80-233 Gdansk, POLAND, e-mail: jas@pg.gda.pl 